\title{
Quantitative EDS of Surface Modified Pd Powders for Hydrogen Storage.
}

Joshua D. Sugar ${ }^{1}$, Mark Homer ${ }^{1}$, Paul G. Kotula ${ }^{2}$, Patrick J. Cappillino ${ }^{3}$, Markus Ong ${ }^{4}$, and David B. Robinson ${ }^{1}$

1. Sandia National Laboratories, Livermore, CA, USA.

2. Sandia National Laboratories, Albuquerque, NM, USA.

3. University of Massachusetts Dartmouth, Chemistry and Biochemistry Department, North Dartmouth, MA, USA.

${ }^{4}$ Whitworth University, Physics Department, Spokane, WA, USA.

Palladium and its alloys are known to be useful for applications such as catalysis [1], electrocatalysis [2], and hydrogen isotope storage and separation [3, 4]. Atomic-scale surface and subsurface layers of other metals have surface hydride energetics that may enhance the kinetics of hydrogen absorption and desorption [5]. In addition, increasing the surface area/volume ratio by making $\mathrm{Pd}$ alloys nanoporous enhances surface-limited reaction rates. When $\mathrm{Pd}$ is alloyed with a higher melting temperature metal such as $\mathrm{Rh}$, the temperature range over which the nanoporous structure remains morphologically stable is extended [6]. We use a variety of techniques to fabricate surface modified Pd powders at different length scales, including, the consolidation of dendrimer-encapsulated nanoparticles [7], surfactant templates [8], and atomic layer electroless deposition (ALED) [9]. It is crucial to understand the spatial distribution of alloying elements in these structures because of the large effect it has on thermal stability, hydrogen storage properties, and the kinetics of hydrogen uptake and release.

Quantitative EDS was performed on Rh and Pt coatings applied to Pd powders at several length scales. Surface-modified powder particles with $5 \mathrm{~nm}$ diameter were fabricated using dendrimer encapsulation [10], 100-nm diameter particles were fabricated with surfactant templates [11], and ALED was used on powders $>1 \mu \mathrm{m}$ in diameter [9]. In all cases, the same analysis routines that utilize a combination of multivariate statistical analysis and multiple-least squares fitting was used to quantify the composition and thickness of the surface modified layer(s). We will discuss the importance of large-area SDD EDS detectors for acquiring data on these surface modified layers that range from tens of nm to sub-nm dimensions.

\section{References:}

1] S. Tanaka, et al., Chemical Communications 47 (2011)

[2] M. Watanabe and S. Motoo, Journal of Electroanalytical Chemistry 60 (1975)

[3] R. Lasser and K.H. Klatt, Physical Review B 28 (1983)

[4] M. Li and W.F. Yang, International Journal of Hydrogen Energy 34 (2009)

[5] J. Greeley and M. Mavrikakis, Journal of Physical Chemistry B 109 (2005)

[6] D.B. Robinson, et al., International Journal of Hydrogen Energy 35 (2010)

[7] R.W.J. Scott, et al., Journal of Physical Chemistry B 109 (2005)

[8] D.B. Robinson, et al., International Journal of Hydrogen Energy 34 (2009)

[9] P.J. Cappillino, et al., Langmuir 30 (2014)

[10] P.J. Cappillino, et al., Journal of Materials Chemistry 22 (2012)

[11] M.D. Ong, et al., Chemistry of Materials 24 (2012)

[12] Sandia National Laboratories is a multi-program laboratory managed and operated by Sandia Corporation, a wholly owned subsidiary of Lockheed Martin Corporation, for the U.S. Department of Energy's National Nuclear Security Administration under contract DE-AC04-94AL85000. 


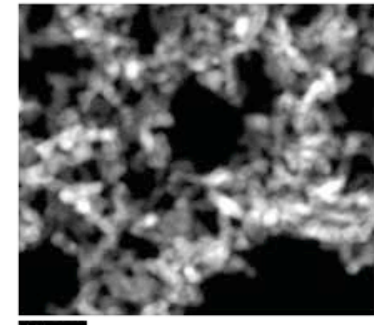

$20 \mathrm{~nm}$

(a)

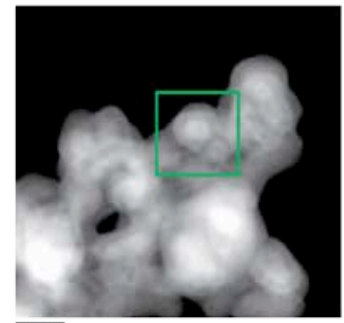

$\mathrm{nm}$

(b)

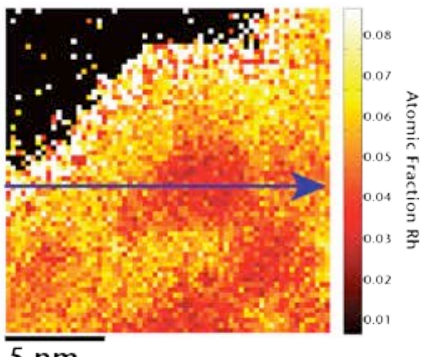

(c)

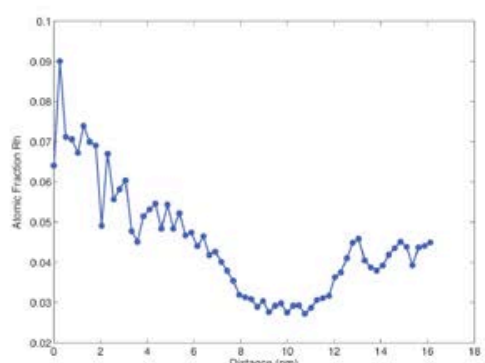

(d)

Figure 1: (a) Lower magnification HAADF STEM image of $5 \mathrm{~nm}$-diameter Pd particles made with dendrimer encapsulation. The green-boxed region in (b) is selected for EDS analysis. The Rh concentration is mapped in (c), and a line profile for the region marked with a blue arrow is shown in (d). The core-shell compositional structure of the particles is visible in the Rh concentration map.

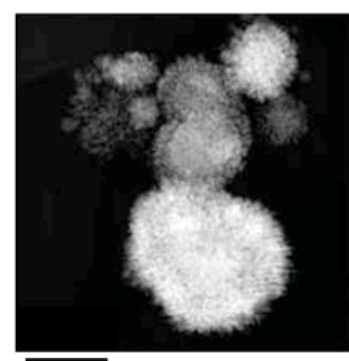

$00 \mathrm{~nm}$

(a)

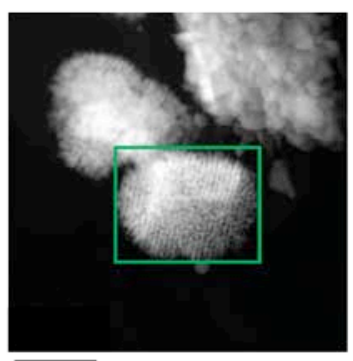

$100 \mathrm{~nm}$

(b)

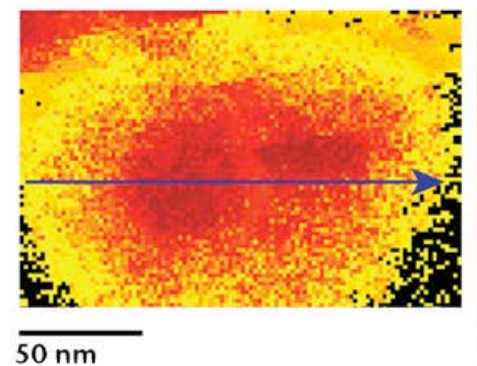

(c)

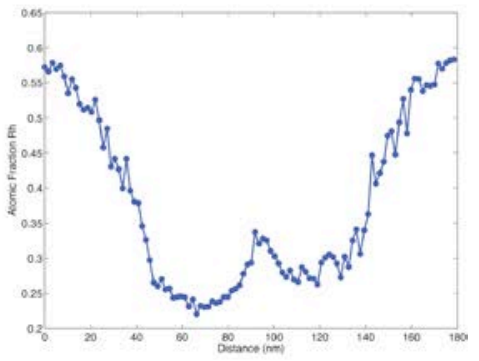

(d)

Figure 2: (a) A HAADF STEM image shows several nanoporous, $100 \mathrm{~nm}$-diameter Pd particles made with surfactant templates. The green-boxed region in (b) is selected for EDS analysis. The Rh concentration is mapped in (c), and a line profile for the blue arrow is shown in (d). Again, the core-shell compositional structure of the particles is visible.

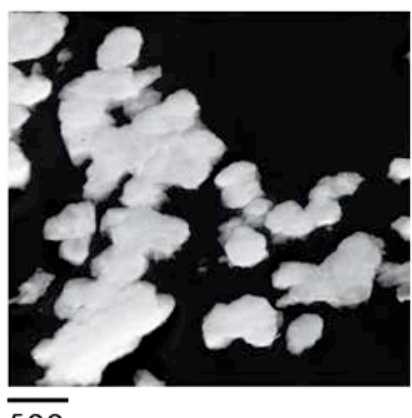

(a)

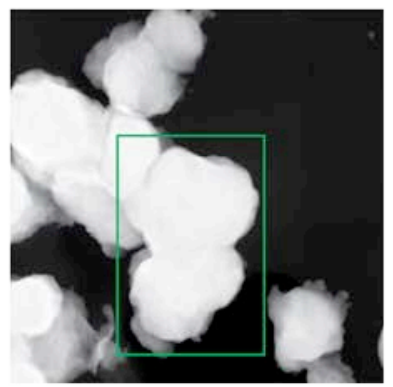

$\overline{200} \mathrm{~nm}$

(b)

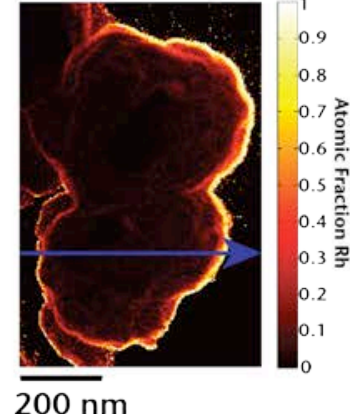

(c)

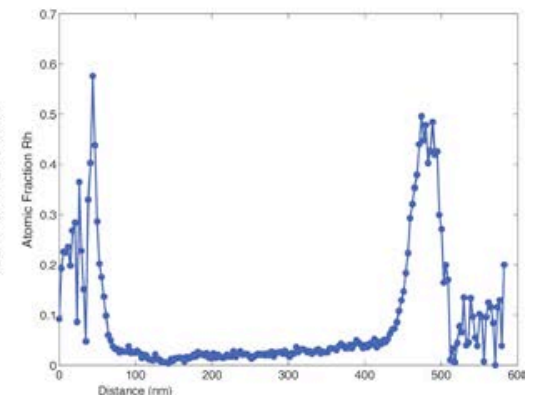

(d)

Figure 3: (a) HAADF STEM image of $\sim 1 \mu \mathrm{m}$-diameter Pd particles that have been surface modified with ALED. These samples were prepared using ultramicrotomy. The green-boxed region in (b) is selected for EDS analysis. The Rh concentration is mapped in (c), and a line profile for the blue arrow is shown in (d). The surface enrichment in Rh is visible. 